Original Article (short paper)

\title{
Effects of extrinsic feedback on the motor learning after stroke
}

\author{
Marcos Antonio Arlindo Soares ${ }^{1}\left[\right.$, Giordano Marcio Gatinho Bonuzzi ${ }^{1,3}$, Daniel Boari Coelho ${ }^{2}$, \\ Camila Torriani-Pasin' ${ }^{10}$ \\ ${ }^{1}$ Universidade de Sao Paulo, USP, Escola de Educacao Fisica e Esporte, Laboratory of Motor Behavior \\ (LACOM), Sao Paulo, SP, Brazil; ${ }^{2}$ Universidade do ABC, Sao Bernardo do Campo, Biomedical \\ Engineerring , Sao Paulo, SP, Brazil, ${ }^{3}$ Universidade Estadual do Piauí, UESPI, Picos, PI, Brazil
}

\begin{abstract}
Aim: This study aims to investigate the effects of the type of extrinsic feedback provided equally in terms of timing and frequency of delivery on the motor learning after stroke. Methods: Twenty post-stroke individuals were distributed randomly into two groups according to the type of feedback provided: knowledge of performance experimental group (KPEG) and knowledge of results experimental group (KREG). Additionally, a control group (CG) was consisted of 20 healthy individuals age- and feedback-matched with the experimental groups. The task was a pointing skill performed in a virtual reality system. The acquisition phase consisted of 3 days/ 75 trials per day. Two retention tests (ret1 and ret2) were run after four days from the acquisition phase. Dependent variable measures were defined by motor performance and movement pattern. Results: The statistical analysis showed interaction effect $\mathrm{F}(3,108=49.13, p=0.01)$ among KPEG and KREG. Based on the motor performance parameters (score) the KPEG improved performance significantly from pre to post $(p=0.001)$, and maintain it from pre to ret1 $(p=0.002)$, and from the pre to ret2 $(p=0.001)$. However, the KREG only showed a difference in motor performance from the pre to post $(p=0.003)$. Compared to the KREG, the KPEG showed improvement on the movement pattern based on the smoothness $(p=0.004)$, which suggests that the KPEG performed more corrections of movements in relation to the CG and KREG groups. Conclusion: These findings suggest that KP allowed better motor learning in individuals after stroke.
\end{abstract}

Keywords: extrinsic feedback; motor learning, stroke.

\section{Introduction}

Several studies have demonstrated the provision of extrinsic feedback induces better conditions for motor learning in individuals after stroke $\mathrm{e}^{1-3}$. The explanation for that may be associated with the possibility of overcoming the exteroceptive and proprioceptive deficits which are frequently seen after stroke $^{4-6}$. This fact makes relevant the offering of extrinsic feedback for this population during motor skill acquisition?

Extrinsic feedback consists of information about movement from an external source. This information can be characterized into two types: knowledge of results (KR), which refers to information provided to the subject about the result of his or her action in relation to the environmental goal; and knowledge of performance (KP), which refers to information provided to the subject regarding the execution pattern of the movement to reach a goal ${ }^{8}$.

Currently, research indicates that sensorimotor performance of the hemiparetic upper extremity after stroke is optimized when different types of extrinsic feedback are used in combination (KP plus KR $)^{9-11}$. However, there are few studies comparing the effects of KP and KR separately on motor learning in individuals after stroke. These investigations presented methodological limitations regarding the different frequency and timing of delivery of feedback, making it difficult to conclude the most appropriate type of feedback on motor learning of these subjects ${ }^{1-3,7}$. Thus, it is crucial to offer the information equally in terms of frequency and timing of delivery, being one group that only receives information about KP and the other group only receives information about KR during the practice of a motor task ${ }^{2}$.

Therefore, the purpose of this study was to investigate the effects of the type of extrinsic feedback provided equally in terms of timing and frequency of delivery on the motor learning of a pointing task in individuals after stroke. We hypothesized that KP will be more effective for improving performance in a pointing task in individuals after stroke because the content of the information is more detailed and may facilitate motor learning through practice.

\section{Materials and Methods}

The experimental procedure was approved by the University of São Paulo's School of Physical Education and Sport's ethics committee, with the number 48605315.9.0000.5391. All the participants signed the term of consent after receiving all the information related to the study. 


\section{Subjects}

Twenty chronic individuals after unilateral stroke (age $=61.3 \pm 10$ years) were included in this study. After the eligibility criteria, the blind assistant researcher randomized the participants and assigned them into the experimental groups according to the type of feedback provided: Knowledge of Performance Experimental Group (KPEG) and Knowledge of Results Experimental Group (KREG), according to computergenerated random assignments. Similarly, twenty healthy age/feedback-matched subjects were recruited to compose the control group (CG) and assigned into one of the groups: Knowledge of Performance Control Group (KPCG) and Knowledge of Results Control Group (KRCG).

The participants were recruited by a convenience sample, from the Rehabilitation Stroke Group at the School of Physical Education and Sports, São Paulo, Brazil.

The inclusion criteria were; 1 - hemiparesis resulting from a single stroke confirmed by neuroimage exams, 2 - time of stroke of at least 6 months, 3 - muscle strength (anterior deltoid, biceps and triceps) on the paretic limb of $\geq 3$ according to the Medical Research Council Scale ${ }^{12}$, and 4 - capacity to understand verbal commands and to perform the task.

The exclusion criteria were more than 5.3 points on the Orpington Prognostic Scale ${ }^{13,14}$, dementia detected by MiniMental State Exam adapted by educational level ${ }^{15}$, limited range of motion with joint impairment in upper limb measured by the passive movement of each joint, comprehension aphasia that prevents understanding of the task, spasticity higher than 2 on the wrist flexors and finger flexors according to the Ashworth Modified Scale ${ }^{16}$, shoulder flexion range of motion less than 45 degrees measured by the active movement with goniometer, painful shoulder reported by the patient in rest or movement (passive or active), lack of visual and auditory acuity with no correction from aid devices, and neglect according to the Star Cancellation Test ${ }^{17}$.

Sample characterization was performed using the following assessments: Orpington Prognostic Scale, Mini-Mental State Exam, Fugl-Meyer Motor Assessment Scale (scores of upper extremity sessions, proprioception, exteroception $)^{18}$, Berg Balance Scale ${ }^{19}$ and Edinburgh Inventory ${ }^{20}$. This last variable was also controlled on the control group in order to avoid the effects of manual dominance. Additional information was collected regarding the medical documents of each patient, such as type of the stroke, side of the lesion, time since lesion, age, gender, and educational level. All evaluations were performed by the blinded assistant researcher.

\section{Instruments and Tasks}

The task consisted of a pointing task that was developed using the STABLE (Motek Force Link ${ }^{\circledR}$ ) equipment. STABLE contains software (D-Flow) in which the task was built. In our task, a target was developed and shown on the screen in the virtual environment. This target was composed of 6 scoring areas (SA), and it has a total diameter of $82 \mathrm{~cm}$. The thickness of each SA was $7 \mathrm{~cm}$. The SA 1 (central) was 12 $\mathrm{cm}$ in diameter. The scores were established from the external scoring area to the internal scoring area as follows: SA $6=1$ point; SA $5=2$ points; SA $4=3$ points; SA $3=4$ points, SA $2=5$ points, and SA $1=6$ points. If the participant reached the area outside the target, the score was 0 points. This score organization was adapted from previous studies that used tasks involving targets ${ }^{21-23}$.

\section{Design and procedures}

All participants performed pointing movements with the hemiparetic arm to achieve the center of the target as accurately as possible. The pointing movements were performed with the vision occluded. This procedure was adopted to avoid corrections in the movement trajectory by the visualization of the target and to optimize the use of proprioceptive information ${ }^{24}$. All the experimental procedures involving the execution of the task were coordinated by the principal investigator.

The experimental procedure established for the task was: 1) Target view (start): The subjects were positioned in front of the virtual reality screen, they remained standing with their arms resting along the body. Then, after the researchers emitted an auditory signal (e.g. "pay attention to the screen"), the participant visualized the target with the message "Observe the target." 2) Three seconds after the target view phase, the target disappeared from the projection screen and a "beep" sounded together was offered together with the appearance of the word start on the projection screen. The subjects closed their eyes and started the pointing movement toward the center of the target in a single trial with their vision occluded. 3) Waiting time: The subjects remained with their arms in the final position that he or she judges to be at the center of the target. At that moment, the participants opened their eyes and visualized the word wait on the projection screen. 4) Providing extrinsic feedback (finish): One second after the previous phase, the final position of their index finger in relation to the target was provided for all subjects, followed by the provision of the respective feedback type (KP or KR).

In the first and fourth stages, the experimental and control design was different, according to the feedback provision (KP or KR). In the first stage of the KP mode, the target was shown on the screen without a score, and in the fourth stage, the trajectory of the movement through a line was demonstrated in relation to the central SA. In the KR mode of the task, in the first stage, the target with the respective score that would be reached in each SA was demonstrated, and in the fourth stage, the respective score reached by the participant was provided. The beginning and the end of both tasks (initial position and final position) can be observed in figures 1 and 2. 
A

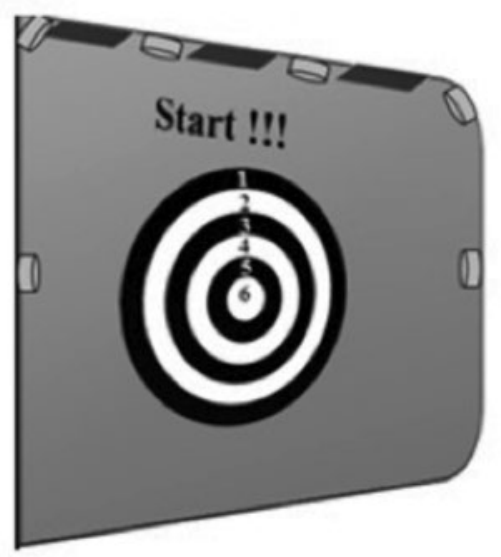

B

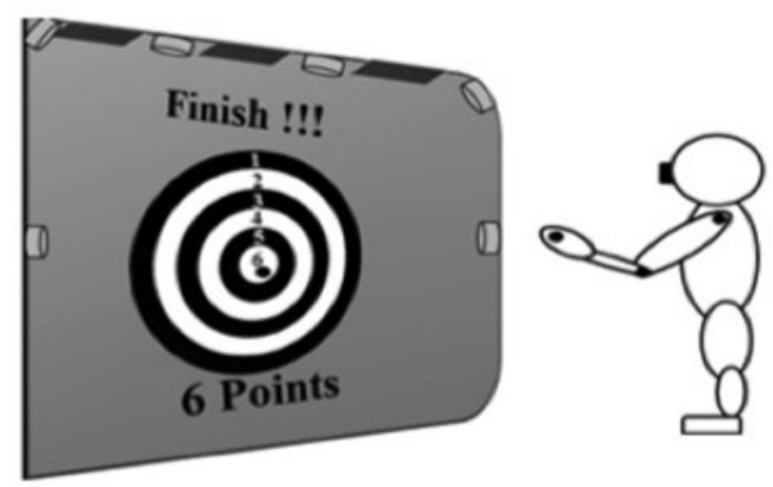

Figure 1. Task knowledge of results, (A) initial position, (B) final position.

A

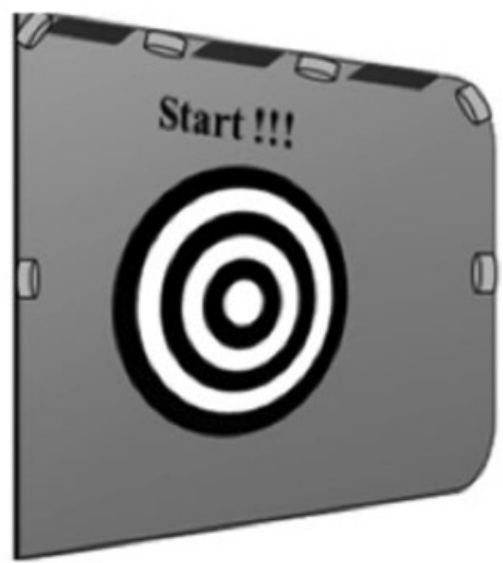

B

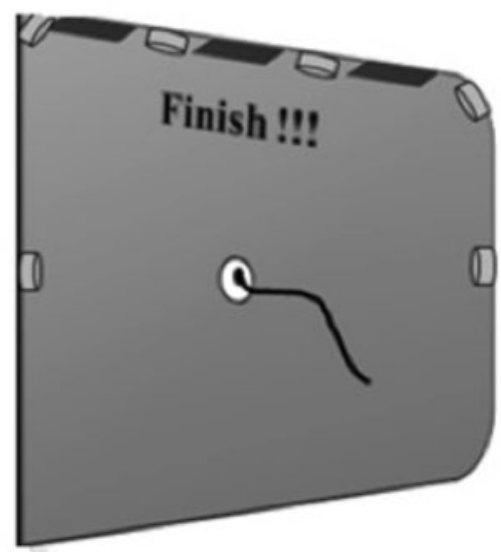

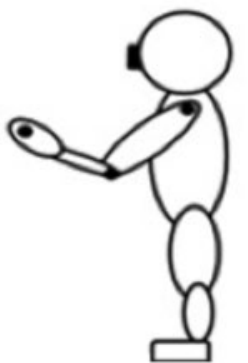

Figure 2. Task knowledge of performance, (A) initial position, (B) final position.

For the kinematic inferences, three reflective markers were fixed on the hemiparetic arm (or corresponding arm in the control group) of all participants: 1) distal phalange of the index finger, 2) ulnar lateral epicondyle, and 3 ) acromial prominence. Then, a splint was used on the paretic limb to keep the hand opened and the wrist neutral, avoiding the increase of tonus on the hand during task performance. In sequence, the participants were positioned, blindfolded, in front of the target at a distance of $1.5 \mathrm{~m}$. The target was placed at a height corresponding to the sternum of the participant. This procedure allowed all pointing movements to be performed at a $45^{\circ}$ shoulder flexion to reach the centre of the target, adjusted according to each participant's height.

The experimental setup was composed of eight days. On the first day, all participants received instructions about the task, and the researcher performed five trials to demonstrate the task. Then, all participants performed three trials as a familiarization phase. After these procedures, the acquisition phase was composed of 225 trials, which was performed during 3 days/ 75 trials per day. Two retention tests (ret1 and ret2) were performed after 4 days from the last acquisition day. Both retention tests were consisted of one block of five trials without feedback and were separated for 10 minutes of rest to avoid fatigue effects. The experimental design can be seen in figure 3 .

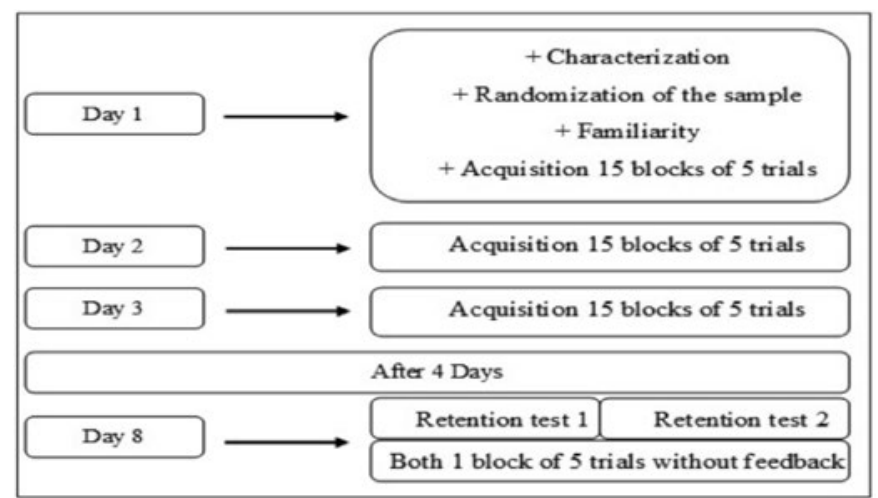

Figure 3. Representative line of the study 
The delivery of extrinsic feedback was equal for all participants in terms of frequency ( $20 \%$ of total trials), referring only to the fifth (last) trials of each block. In terms of timing, feedback was provided in a terminal way, with a delay of three seconds after the last trial was completed.

\section{Measures}

Our dependent variables involved assessments related to motor performance and movement patterns.

The measures of motor performance were: A) Score derived from the subject's performance in relation to the reached in each trial. B) Linearity given by the ratio of the distance between the initial marker position and the target by the length of hand displacement in the 3D movement expressed in a nondimensional way. C) Peak velocity on finger $(\mathrm{m} / \mathrm{s})$ defined as the moment of the maximum velocity of the finger marker; shoulder-to-elbow angle, from the ratio between the angle of the shoulder (formed from the displacement of the elbow reflective marker in relation to the shoulder marker observed in a straight line in the sagittal plane).

The measures of movement patterns were: A) Movement time(s) defined as the total time in which the individual performed the action, considering movement initiation/end as the time at which the finger achieved the velocity of $20 \mathrm{~mm} / \mathrm{s}$ with increasing/decreasing subsequent values. B) Smoothness defined as a peak between two valleys in the velocity curve with a difference between them greater than $1 \mathrm{~cm} / \mathrm{s}$, expressed in a non-dimensional way. $\mathrm{C}$ ) the angle of the elbow (formed from the angle created between the shoulder and finger markers in relation to the marker of the elbow), expressed in a nondimensional way.

The kinematics of the motor standard measurements were obtained through the reflective markers, recorded at a frequency of $100 \mathrm{~Hz}$, in the $\mathrm{X}, \mathrm{Y}$, and $\mathrm{Z}$ axes. Following visual inspection, the analysis was performed through custom-made MATLAB (Mathworks, Inc, Natick, MA) routines. Raw data were filtered through a dual-pass fourth-order Butterworth filter with a cut-off frequency set at $10 \mathrm{~Hz}$.

\section{Data analysis}

Statistic Software version 11 was used to run inferential analyse. Shapiro-Wilk and Levene's tests confirmed normality and homogeneity, respectively, and a parametric analysis was performed. Differences in characterization measures between the experimental groups were determined using a Student's t-test for independent measures. The motor performance and movement parameters dependent variables were compared between the groups (KPEG x KREG x KPCG x KRCG), comparing pretest (pre), post-test (post), retention test 1 (ret1), and retention test 2 (ret2), using a two-way analysis of variance (ANOVA, 4 groups $\mathrm{x} 4$ periods), with repeated measures in the last factor; followed by the Tukey's post hoc test. The significance level for all statistical comparisons was $5 \%$.

\section{Results}

\section{Participants}

The Student's t-test showed a significant difference between the KPEG and KREG groups in relation to the Fugl-Meyer proprioceptive session $(\mathrm{p}=0.02)$. This result indicates KPEG $($ mean $=6.6)$ being more impaired than the KREG $($ mean $=7.8)$ regarding the proprioceptive deficits resulting from stroke. For all other characterization measures, the t-test did not indicate significant differences, which demonstrates the homogeneity of the groups in relation to the other variables (table 1).

Table 1: Demographic and clinical data of the experimental groups.

\begin{tabular}{|c|c|c|c|c|}
\hline \multirow{2}{*}{ Characteristic } & \multicolumn{2}{|c|}{ Groups } & & \\
\hline & KP & $\mathrm{KR}$ & & \\
\hline Genre (M/F) & $5 / 5$ & $5 / 5$ & & \\
\hline Age (years) & $61.7 \pm 11.5$ & $60.5 \pm 10.6$ & {$[0,30]$} & $(0.55)$ \\
\hline Time since onset (months) & $98.1 \pm 99.2$ & $39.3 \pm 22.3$ & {$[1,82]$} & $(0.08)$ \\
\hline Side of lesion $\mathrm{L} / \mathrm{R}$ & $6 / 4$ & $5 / 5$ & & \\
\hline Type of Stroke I/H & $7 / 3$ & $8 / 2$ & & \\
\hline Educational Level ES/HS/HE & $5 / 1 / 4$ & $3 / 2 / 5$ & & \\
\hline Orpington Prognostic & $3 \pm 0$ & $3 \pm 0$ & & \\
\hline Mini-Mental State Examination & $25.5 \pm 3.2$ & $27.2 \pm 3.5$ & {$[-1,29]$} & $(0.21)$ \\
\hline Fugl-Meyer socre UL (66) & $47 \pm 15.2$ & $52.4 \pm 7.4$ & {$[-1,00]$} & $(0.37)$ \\
\hline Proprioceptive score (16/8) & $6.6 \pm 1.5$ & $7.8 \pm 0.4$ & {$[-2,42]$} & $(0.02)^{*}$ \\
\hline Exteroceptive socre $(8 / 4)$ & $3.5 \pm 0.7$ & $3.5 \pm 0.8$ & {$[-0,53]$} & $(0.59)$ \\
\hline Berg Balance score (56) & $45.6 \pm 9.2$ & $45.4 \pm 7.5$ & {$[-2,98]$} & $(0.95)$ \\
\hline Edinburgh Inventory L/R & $0 / 10$ & $1 / 9$ & & \\
\hline
\end{tabular}

Legend: Side of lesion L/R= left, right; Type of stroke I/H = ischemic, hemorrhagic; Educational level ES/HS/HE = elementary school, high school, higher education; Fugl - Meyer score UL= Upper limb; Edinburgh Inventory L/R = left, right; [ ] = $t$ value ; ( )= $p$ value; * = significant difference. 


\section{Motor performance measurement}

In the analyses of the score, the two-way ANOVA detected interaction effects $F(3,108)=49.13, p=0.001$, and Tukey's post hoc test confirmed a significant difference related to the periods: KPCG presents differences from the pre to post $p=0.001$, from the pre to ret $1 p=0.001$, and from the pre to the ret2 $p=0.001$. The KRCG was different from the pre to ret2 $p=0.001$. The KPEG was different from the pre to post $p=0.001$, from the pre to ret $1 p=0.002$, and from the pre to ret2 $p=0.001$; and the KREG showed a difference only from the pre to post $p=0.003$. These results suggest no differences in the learning process between KPCG, KRCG, and KPEG. All groups were able to increase motor performance in the acquisition phase and maintain it in the retention tests. Only the KREG group was unable to keep performance in retention tests, (Figure 4A).

Regarding linearity (Figure 4B), the two-way ANOVA detected a significant difference in periods $F(3,108)=4.48$, $p=0.005$, and Tukey's post hoc test confirmed a significant difference between the pre and post $p=0.001$ and pre to ret 2 $p=0.001$. It is noted that the movement of all groups became more linear from the pre to post and from the pre to ret2.

In the peak finger velocity, the two-way ANOVA demonstrated a significant difference in the periods $F(3,108)=10.50, p=0.000$, and the Tukey post hoc test revealed that this difference occurred between pre and post $p=0.001$, among pre to ret $1 p=0.002$, and pre to ret $2 p=0.002$. These results demonstrate that the peak velocity was higher in the pre. However, in the post, ret1, and ret2, there was a decrease in the velocity peak (Figure 4C).
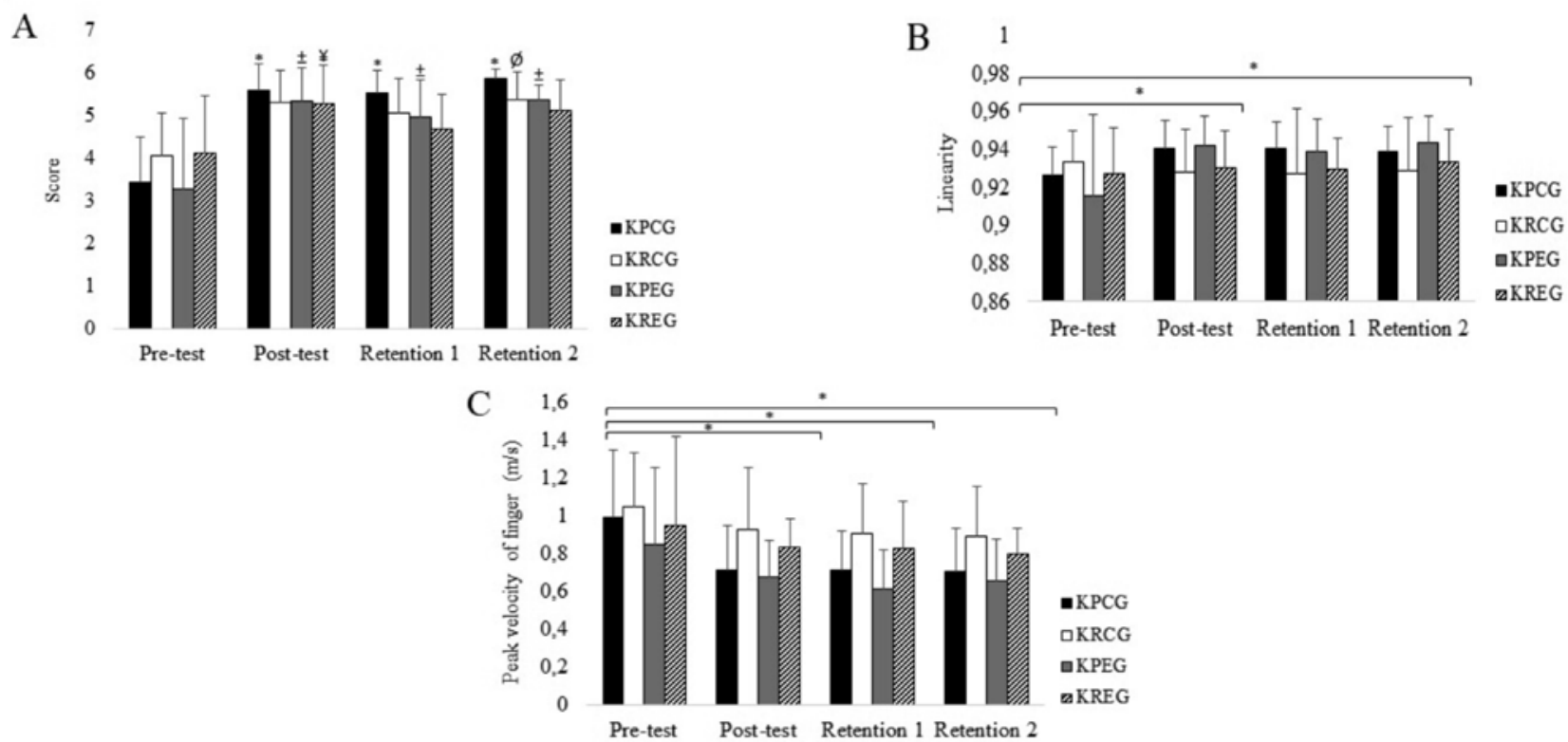

Figure 4A, B, C. KPCG = knowledge of performance control group, KRCG knowledge of results control group, KPEG $=$ knowledge of performance experimental group, $\mathrm{KREG}=$ knowledge of results experimental group. Figure $4 \mathrm{~A}=*=$ difference in the periods factor in relation to the pre-test KPCG, $Q=$ periods difference in relation to the pre-test $\mathrm{KRCG}, \pm=$ periods difference in relation to the pre-test $\mathrm{KPEG}, ¥=$ periods difference in relation to the pre-test KREG. Figure $4 \mathrm{~B}, \mathrm{C}=*=$ significant difference intragroup.

\section{Motor pattern Measurement}

The movement time, through the two-way ANOVA, no significant differences were found for interaction effect $F(3,108)=0.56, p=0.825$, intergroup $F(3,108)=1.73$, $p=0.178$, and periods $F(3,108)=0.45, p=0.716$. These findings may be directly related to the main requirement of the task, which was a maximum precision task (Figure 5A).

Smoothness (Figure 5B), had a significant difference in intergroup $F(3,36)=6.65, \mathrm{p}=0.001$ and periods $\mathrm{F}(3,108)=2.72$, $\mathrm{p}=0.047$, where the Tukey test revealed that this difference occurred between KRCG and KPEG $\mathrm{p}=0.001$ and between KPEG and KREG $\mathrm{p}=0.004$ at all periods. From these results, the KPEG group performed more corrections of movement in relation to the KPCG and KREG groups.

In relation to the angle of shoulder to the elbow, the twoway ANOVA detected a significant difference in periods $\mathrm{F}(3$, $108)=5.06, p=0.002$, and the Tukey post hoc test confirmed a significant difference between pre and post $\mathrm{p}=0.002$, pre to ret 1 $\mathrm{p}=0.004$, and pre to ret $2 \mathrm{p}=0.002$. These results demonstrate a decrease in the use of the shoulder angle in relation to the elbow angle of the pre in relation to the post, ret1, and ret2 (Figure 5C). 

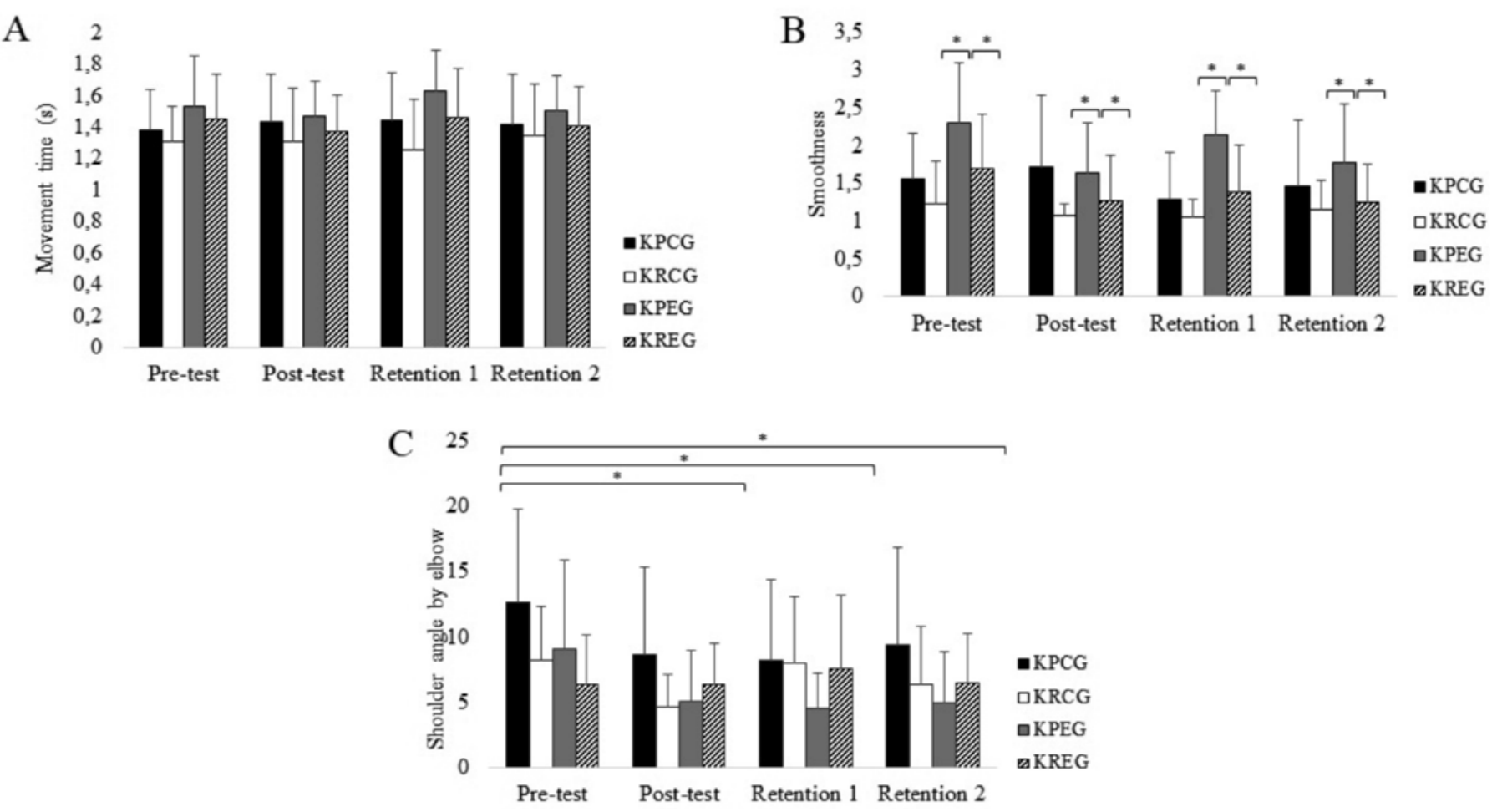

Figure 5A, B, C. KPCG $=$ knowledge of performance control group, $\mathrm{KRCG}=$ knowledge of results control group, KPEG $=$ knowledge of performance experimental group, $\mathrm{KREG}=$ knowledge of results experimental group, Figure $5 \mathrm{~B}=*=$ significant difference intergroup. Figure $5 \mathrm{C}=*$ significant difference intragroup.

\section{Discussion}

Our purpose was to investigate the effects of the type of extrinsic feedback provided equally in terms of timing and frequency of delivery on the motor learning in individuals after a stroke. Thus, we used virtual reality environment because of its feasibility and safety in the performance of the upper extremity after stroke ${ }^{25,26}$, besides it is an easy environment for manipulation of extrinsic feedback. The present study showed that the KPEG was able to improve its performance and keep it in the retention tests, confirming our hypotheses. On the other hand, the KREG presented a significant difference only from pre to post, demonstrating no maintenance of the improved performance in the retention tests.

In our results, the individuals after a stroke who received KR feedback did not show motor learning using their affected upper extremity. The information provided related to the KP seems to be significant and relevant to the individuals after a stroke, because this information may complete the feedback intrinsically that may be impaired after stroke. After a stroke, the individuals may not be aware of their movement patterns while performing a task. This unawareness related to the movement may be derived from impairments in their intrinsic feedback system $^{7}$, making difficult the detection of information captured by sensory receptors. The information provided as KP may be processed as an increment resource for the intrinsic information processing, given that the KR tends to direct the subject to process more external information ${ }^{8}$.
Our results may also suggest that after a stroke the individual who received KR were less accurate than those who received KP. These results are contrary to the results presented by Cirstea, Ptito and $\operatorname{Levin}^{27}$ and Cirstea and Levin ${ }^{28}$, in which the supply of KR was superior to the KP in relation to accuracy. Possible explanations for our results may be related to the proprioceptive deficits presented by the KPEG. Those deficits made the provision of information related to movement pattern relevant to the execution of a more coordinated movement of reaching with greater accuracy.

Regarding motor patterns, the results indicated that the variable time of movement did not present significant differences for both EG and CG groups. However, when the peak finger velocity measures are observed, EG and CG became consistent in terms of movement acceleration, suggesting the acquisition of a better motor control along of the practice. Similar results as ours have seemed in other studies with the same task comparing individuals after stroke and healthy subjects. In these studies, pointing movements performed in a virtual environment are slower and less accurate and useless trunk displacement in relation to the physical environment for both populations ${ }^{28,29}$.

Besides, the literature emphasizes that execution of a movement of pointing toward the goal involves the planning of the movement trajectory and specifies interarticular coordination ${ }^{29}$. Both characteristics can be found in the results of the present study, given that both post-stroke and healthy subjects were able to obtain a higher degree of linearity throughout the acquisition and maintained them in the retention phase, which evidences 
more linear movements towards the target and a better endpoint control along of the movement. In addition, the decrease in the use of the shoulder angle and greater use of the elbow angle in individuals after stroke demonstrates a better strategy in terms of coordination to perform the task ${ }^{27,28,30}$.

The results related to smoothness, which demonstrated significant differences between KPEG and KREG, showed that individuals after stroke who received KP information had more movement corrections (less smoothness) than the KR group. According to some authors, deficits in interarticular coordination may be associated with sensory-motor delays that interfere directly with the smoothness of movement ${ }^{29,31}$, and it may induce different conditions of motor control for the KPEG.

In our study, the KPEG presented higher proprioceptive deficits. This impairment may explain the lower smoothness found. Proprioception is one of the sources of sensory information that most influences the control of movement. Therefore, proprioceptive changes can reduce the ability to feel and perceive the location of the position of the limbs' movements in space ${ }^{32-34}$. This limitation may be associated with the lack of precision with respect to internal representations in relation to how the movement was performed. It may be evident that studies investigating the effects of the extrinsic feedback type in post-stroke subjects need to stratify the groups in a balanced way regarding sensorial impairments in order to solve this issue more precisely. Moreover, studies may have to consider conduct a more precise proprioceptive evaluation before proposing learning strategies for skill acquisition.

However, even with more movement corrections, the KPEG was able to obtain better learning in a pointing task than the KREG. This result corroborates results reported in the literature in which the supply of KP is more effective to improve the motor performance $\mathrm{e}^{27,28,35}$. According to these authors, KP provides critical information on how to adapt motor behavior to improve efficiency in subsequent trials. Thus, in individuals after stroke may be needed to offer KP information on how to correct movements. The transmission of this information may help patients plan a movement with upper extremity and achieve the task learning, especially when these subjects present proprioceptive deficits, as observed in our study.

One possible limitation of our study could be the heterogeneity of characteristics in relation to the proprioceptive systems in the EG. Another limitation was not to control the velocity of the arm during the task performance. It is simply possible that the subjects were using a speed-accuracy tradeoff, where they sacrificed speed for better accuracy. To the best of our knowledge, this is the first study that points out the necessity of precisely evaluate and consider proprioceptive as a variable for randomization procedure. Future research may have to consider the randomization of EG, taking into account the sensory impairments of individuals post-stroke.

\section{Conclusion}

In summary, the provision of KP was more effective than KR for motor learning of a pointing task performed with the paretic limb for individuals after stroke. The KP provided better control of endpoints and better intersegment coordination. Interventions may have to consider sensory impairments as a limitation for the use of KR in order to facilitate motor learning. Explicit feedback is a feasible strategy to be part of the planning interventions aimed at improving the performance of the upper extremity after stroke.

\section{References}

1. Dijk H Van, Jannink MJA, Hermens HJ. Effect of augmented feedback on motor function of the affected upper extremity in rehabilitation patients: A systematic review of randomized cotrolled trials. J Rehabil Med. 2005;37:202-11.

2. Molier BI, Van Asseldonk EHF, Hermens HJ, Jannink MJA. Nature, timing, frequency and type of augmented feedback; does it influence motor relearning of the hemiparetic arm after stroke? A systematic review. Disabil Rehabil. 2010;32(22):1799-809.

3. Subramanian SK, Massie CL, Malcolm MP, Levin MF. Does provision of extrinsic feedback result in improved motor learning in the upper limb poststroke? A systematic review of the evidence. Neurorehabil Neural Repair. 2010;24(2):113-24.

4. Bagesteiro LB, Sarlegna FR, Sainburg RL. Differential influence of vision and proprioception on control of movement distance. Exp brain Res. 2006 May;171(3):358-70.

5. Vidoni ED, Boyd LA. Preserved motor learning after stroke is related to the degree of proprioceptive deficit. Behav Brain Funct. 2009; 10:1-10.

6. Cumming TB, Marshall RS, Lazar RM. Stroke, cognitive deficits, and rehabilitation: still an incomplete picture. Int J Stroke. 2013; 8(1):38-45.

7. Van Vliet PM, Wulf G. Extrinsic feedback for motor learning after stroke: what is the evidence? Disabil Rehabil. 2006; 28(13-14):831-40.

8. Schmidt R.A, Lee TD. Motor control and learning: a behavioral emphasis. Champaign: Human Kinetics; 2014.

9. Dancause N, Ptito A, Levin MF. Error correction strategies for motor behavior after unilateral brain damage: short-term motor learning processes. Neuropsychologia. 2002;40:1313-23.

10. Maulucci RA, Eckhouse RH. Retraining reaching in chronic stroke with real-time auditory feedback. NeuroRehabilitation. 2001;16(3):171-82.

11. Gilmore PE, Spaulding SJ. Motor Control and Motor Learning : Implications for Treatment of Individuals Post Stroke. Phys Occup Ther Geriatr. 2001; 20(1):1-15.

12. McCluskey D. Aids to the examination of the peripheral nervous system. Ulster Med J. 1989;58(45):201.

13. Kalra L, Crome P. The Role of Prognostic Scores in Targeting Stroke Rehabilitation in Elderly Patients. J Am Geriatr Soc. 1993; 41(4):396-400.

14. Lai SM, Duncan PW, Keighley J. Prediction of functional outcome after stroke: comparison of the Orpington Prognostic Scale and the NIH Stroke Scale. Stroke. 1998;29(9):1838-42.

15. Folstein MF, Folstein SE, McHugh PR. "Mini-mental” A practical method for grading the cognitive state of patients for the clinician. J Psychiatr Res. 1975; 12(3):189-98. 
16. Bohannon RW, Smith MB. Interrater reliability of a modified ashworth scale of muscle spasticity. Class Pap Orthop. 1987;67:415-7.

17. Wilson B, Cockburn J, Halligan P. Development of a behavioral test of visuospatial neglect. Arch Phys Med Rehabil. 1987; 68(2):98-102.

18. Fugl-Meyer AR, Jääskö L, Leyman I, Olsson SSS. The post-stroke hemiplegic patient. 1. a method for evaluation of physical performance. Scand J Rehabil Med. 1975;7:13-31.

19. Berg K. Measuring balance in the elderly: Development and validation of an instrument. Can J Public Heal. 1992; 83:1-130.

20. Oldfield RC. The assessment and analyses of handedness: Edinburgh inventory. Neuropsychologi. 1971; 97-113.

21. Meira Junior CM, Perez CR, Maia RF, Neiva JFO, Barrocal RM. Extroversão, neuroticismo e desempenho motor em crianças executando arremessos de dardo de salão. Rev Bras Psicol do Esporte. 2008;2(1):1-14.

22. Meira Junior CM, Tani G. the Contextual interference effect in acquisition of dart-throwing skill tested on a transfer test with extended trials. Percept Mot Skills. 2001;92(3):910-8.

23. Corrêa UC, Benda RN, Tani G. Estrutura De Prática E Processo Adaptativo Na Aquisição Do Arremesso De Dardo De Salão. Rev Bras Ciência do Esporte. 2001;22:69-83.

24. Cirstea MC, Levin MF. Compensatory strategies for reaching in stroke. Brain. 2000;123(5):940-53.

25. Crosbie J, Lennon S, McGoldrick M, McNeill M, McDonough S. Virtual reality in the rehabilitation of the arm after hemiplegic stroke: a randomized controlled pilot study. Clin Rehabil. 2012;26(9):798-806.

26. Yin CW, Sien NY, Ying LA, Chung SF-CM, Tan May Leng D. Virtual reality for upper extremity rehabilitation in early stroke: a pilot randomized controlled trial. Clin Rehabil . 2014;28(11):1107-14.

27. Cirstea CM, Ptito A, Levin MF. Feedback and cognition in arm motor skill reacquisition after stroke. Stroke. 2006; 37(5):1237-42.

28. Cirstea MC, Levin MF. Improvement of arm movement patterns and endpoint control depends on type of feedback during practice in stroke survivors. Neurorehabil Neural Repair. 2007; 21(5):398-411.

29. Levin MF. Interjoint coordination during pointing movements is disrupted in spastic hemiparesis. Brain. $1996 ; 119$ (1):281-93.

30. Cirstea MC, Ptito A, Levin MF. Arm reaching improvements with short-term practice depend on the severity of the motor deficit in stroke. Exp Brain Res. 2003;152:476-88.

31. Schaefer SY, Hengge CR. Testing the concurrent validity of a naturalistic upper extremity reaching task. Exp Brain Res. 2016;234(1):229-40.
32. Findlater SE, Dukelow SP. Upper Extremity Proprioception After Stroke: Bridging the Gap Between Neuroscience and Rehabilitation. J Mot Behav. 2016;1-8.

33. Magill RA. Aprendizagem Motora: conceitos e aplicações. 5th ed. São Paulo: Edgard Blücher; 2000.

34. Sarlegna FR, Sainburg RL. The Roles of Vision and Proprioception in the Planning of Reaching Movements. Adv Exp Med Biol. 2013;317-35.

35. Sharma D. Knowledge of Result Versus Knowledge of Performance in Learning Motor Skilled Activity for Upper Limb in Recovery Stage of Stroke . IJSR - Int J Sci Res. 2016; 5:3-6.

\section{Acknowledgments}

The authors wish to thank: all the subjects and their parents for their participation and commitment to the study; Rubiel Barrera, Neal Salamanca, Stephania Moreno, Eduardo Sarmiento, and all the student members of the Human Movement Sciences Research Group; and Los Llanos University's Department of Physical Education and Faculty of Education. This study was supported by the Los Llanos University Research Department.

\section{Corresponding author}

Marco Antonio Arlindo Soares

Universidade de Sao Paulo, USP, Sao Paulo, Brazil

Email: srs.marcos@hotmail.com

Manuscript received on June 12, 2018

Manuscript accepted on October 10, 2018

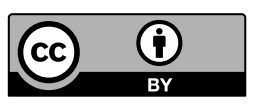

Motriz. The Journal of Physical Education. UNESP. Rio Claro, SP, Brazil - eISSN: 1980-6574 - under a license Creative Commons - Version 4.0 